SMASIS2014-7581

\title{
PIEZO-OPTICAL ACTIVE SENSING WITH PWAS AND FBG SENSORS FOR STRUCTURAL HEALTH MONITORING
}

\author{
Bin Lin, Victor Giurgiutiu \\ University of South Carolina \\ Columbia, SC 29208, USA \\ linbin@cec.sc.edu
}

\begin{abstract}
This paper presents the investigation of piezo-optical active sensing methodology for structural health monitoring (SHM). Piezoelectric wafer active sensors (PWAS) have emerged as one of the major structural health monitoring (SHM) technology; with the same installation of PWAS transducers, one can apply a variety of damage detection methods; propagating acousto-ultrasonic waves, standing waves (electromechanical impedance) and phased arrays. In recent years, fiber Bragg gratings (FBG) sensors have been investigated as an alternative to piezoelectric sensors for the detection of ultrasonic waves. FBG have the advantage of being durable, lightweight, and easily embeddable into composite structures as well as being immune to electromagnetic interference and optically multiplexed.

In this paper, the investigation focused on the interaction of PWAS and FBG sensors with structure, and combining multiple monitoring and interrogation methods (AE, pitch-catch, pulseecho, phased-array, thickness mode, electromechanical impedance). The innovative piezo-optical active sensing system consists of both active and passive sensing. PWAS and FBG sensors are bonded to the surface of the structure, or are integrated into structure by manufacturing process. The optimum PWAS size and excitation frequency for energy transfer was determined. The FBG sensors parameters (size, spectrum, reflectivity, etc.) for ultrasonic guided waves sensing were also evaluated. We focused on the optimum FBG length and design to improve the sensitivity, coverage, and signal to noise ratio. In this research, we built the fundamental understanding of different sensors with optimum placement. Calibration and performance improvements for the optical
\end{abstract}

interrogation system are also discussed. The paper ends with conclusions and suggestions for further work.

\section{INTRODUCTION}

Structural health monitoring (SHM) is an area of growing interest and worthy of new and innovative approaches. The increasing age of our existing infrastructure makes the cost of maintenance and repairs a growing concern. Structural health monitoring may alleviate this by replacing scheduled maintenance with as-needed maintenance, thus saving the cost of unnecessary maintenance, on one hand, and preventing unscheduled maintenance, on the other hand. For new structures, the inclusion of structural health monitoring sensors and systems from the design stage is likely to greatly reduce the life-cycle cost. The key technology to an effective SHM system is the sensing element. The past two decades have witnessed an extensive development of SHM sensor technology [1]-[3]. A wide range of sensors have been developed particularly for generating and receiving acousto-ultrasonic waves. Common examples of such SHM sensors are the piezoelectric wafer active sensor (PWAS) transducers [4] and the fiber Bragg grating (FBG) optical sensors [5]. The PWAS transducers serve as both transmitters (exciters) and receivers (sensors) of structural guided waves, whereas the FBG sensors can only act as receivers. (Recent developments have also achieved the generation of guided waves with FBG sensors through the thermo-optical effect[6], but the resulting amplitudes are still order of magnitude below those achieve with piezoelectric transducers). 


\section{Piezoelectric Wafer Active Sensors}

PWAS have emerged as one of the major SHM technologies. A variety of damage detection methods can be applied to this type of sensor [1]: (a) propagating ultrasonic waves, both acoustic emission (AE) and guided ultrasonic waves (GUW); and (b) standing ultrasonic waves, i.e., electromechanical impedance spectroscopy (EMIS) as illustrate in Fig 1 and Fig 2.

\section{Propagating Lamb waves}

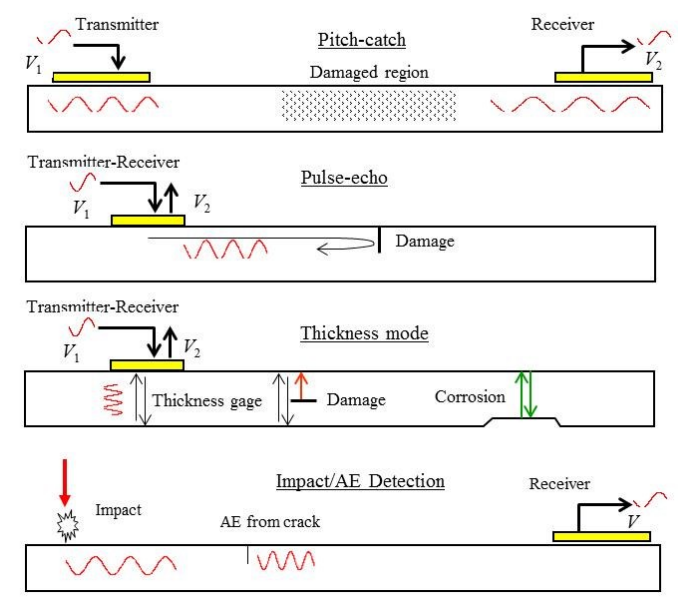

Fig 1: The PWAS are used for structural sensing with propagating ultrasonic guided waves. The propagating wave methods include: pitch-catch; pulse-echo; thickness mode; and passive detection of impacts and acoustic emission (AE).
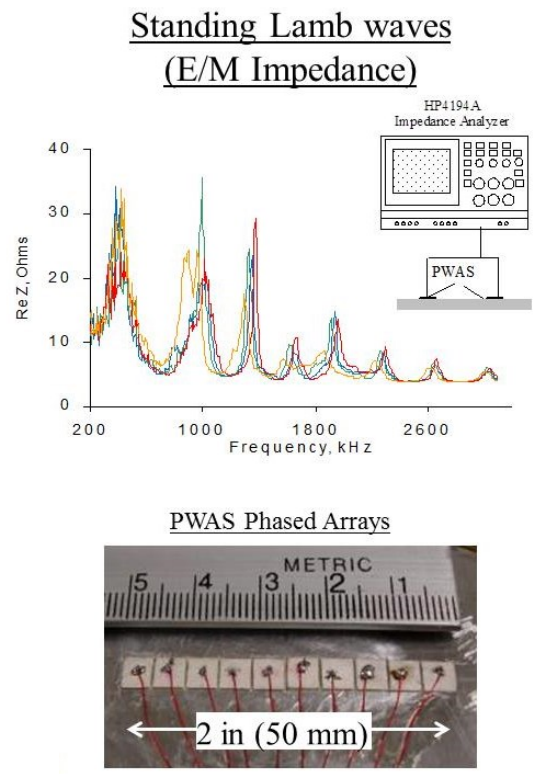

Fig 2: The PWAS are used for structural sensing includes standing waves and phased arrays.
- $\quad$ AE for crack initiation has been shown to enable the detection of crack initiation and crack progression; $\mathrm{AE}$ provides earlier warning of impending damage than any other methods. Because it is very sensitive to damage events, the $\mathrm{AE}$ method has been used for many applications in aerospace and civil engineering applications.

- GUW quantitative damage detection and evaluation relies on in interrogative ultrasonic waves propagating and reflecting within the structure to identify wave field disturbances due structural damage and flaws. An N-SHM system using interrogative GUW would be able to cover large areas from one single location, thereby being cost-effective and time-efficient. Research on embedded PWAS GUW-SHM has been conducted nationally and internationally for damage detection on both metallic and composite thin-walled structures.

- EMIS for local material degradation monitoring: is considered a promising approach for PWAS structural NDE. This method utilizes high frequency structural excitations, which are typically higher than $30 \mathrm{kHz}$ through surface-bonded PWAS to monitor changes in the structural E/M impedance. Previous studies have confirmed that EMIS is sensitive to very small amounts of material changes, suggesting that EMIS offers the potential for detection of the progression of small damage at the material-level in a metallic material.

\section{Fiber Bragg Grating Sensors}

Fiber optics sensors have known extensive development for SHM applications. Optical fibers consist of a very small inner core (which has a high reflection index caused by germanium doping) and an outer part of pure glass with a smaller reflection index. Total internal reflection takes place due to the large difference in the reflection indices. The FBG sensor (Fig 3) is a permanent periodical perturbation (grating) in the index of refraction of the optical fiber core inscribed at selected locations using high-intensity UV light. This periodic perturbation with pitch $\Lambda$ acts as a wavelength filter with a narrowband reflection spectrum centered on the Bragg wavelength $\lambda_{B}=2 \eta_{\text {eff }} \Lambda$ (Fig $3 b$ ).

If the temperature is constant, only the effect of strain is present and the strain and Bragg wavelength relationship simplifies to

$$
\Delta \lambda_{B}=\lambda_{B}\left(1-\rho_{\alpha}\right) \Delta \varepsilon
$$

Thus, an FBG sensor bonded to a structural surface would respond to the structural strain by shifting its spectrum as indicated by Eq. (1).

Fiber optics sensors offer several advantages over piezoelectric sensors for SHM applications: (a) immune to electromagnetic interference (EMI); (b) corrosion resistance; (c) the promise of direct embedment into the composite 
material along with the reinforcing fibers; (d) capability of working in wet and/or underwater environments, etc. In addition, FBG sensors offer the possibility of multiplexing several sensors of slight different wavelength on the same optical fiber and interrogating them individually. Fiber optics sensors also have several limitations that have impeded their widespread usage: (i) the need for considerable optoelectronic equipment to convert the optical changes into actual readings of the physical quantity being monitored (strain, or other material property); (b) bandwidth limited by the bandwidth of the optoelectronic equipment that has to perform complicated processing of the optical signal; etc. The methods used for the demodulation and interpretation of the optical signal are very diverse and still evolving.

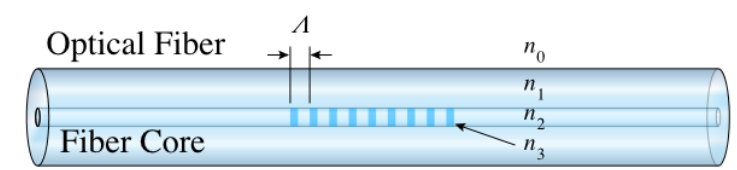

(a)

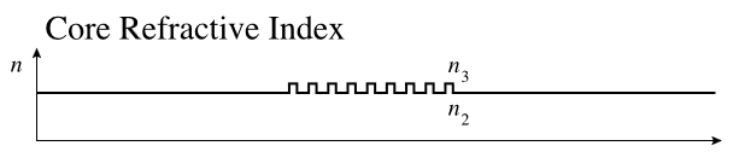

(b)
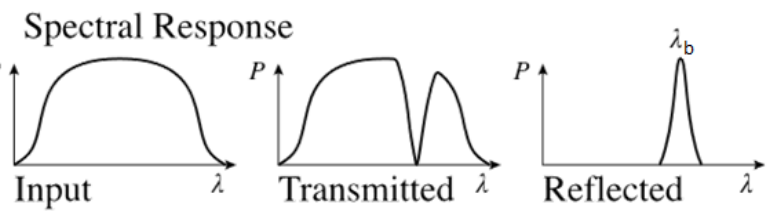

Fig 3 (a) Principles of fiber Bragg grating (FBG) optical sensors; (b) details showing the notch in the transmission spectrum and the peak in the reflection spectrum at the Bragg wavelength

One common way of using the FBG sensor for strain measurements is to track the spectral shift $\Delta \lambda_{B}$ of the reflected signal and convert it into strain change $\Delta \varepsilon$ according to Eq.(1). However, this type of demodulation is only effective for sizable strain values (say, several $\mu \varepsilon$ ) and is not effective for the very small strains encountered in ultrasonics wave propagation which are several order of magnitude smaller ( $0.010-0.100 \mu \varepsilon)$. The demodulation method used for the detection of such small strains is based on up and down excursions from the midpoint of spectral slope (Fig 4). This socalled FWHM (full-width half-maximum) method uses a narrow-band tunable laser source precisely positioned on the FWHM point of the spectrum and several optical components to direct the reflected optical signal to a low-noise photo detector where is converted into an electrical signal that can be fed into an oscilloscope for display and digitization.

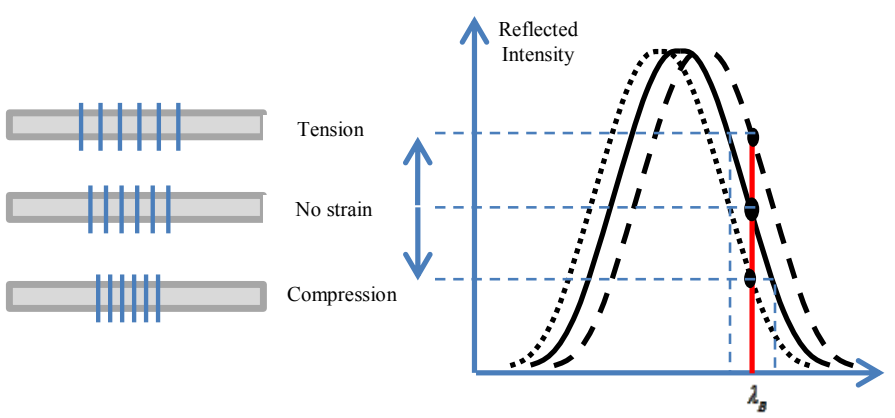

Fig 4: Principle of an operation of intensity-based full-width half-maximum (FWHM) FBG interrogation system [7]

In previous study, an automated optical FBG FWHM intensity system with accurate nano-strain reading at high sampling rate (up to $5 \mathrm{MHz}$ ) has been developed for ultrasonic guided wave SHM [7]. The literature review showed that there is no commercially available product for detection of ultrasonic guided waves because: (a) the frequency is high; (b) the strains are very small (nano-strain). The previous research focused on the FWHM intensity method and the optical hardware selection and optimization to increase both the sensitivity and speed of the FWHM system. The selection of TLS source, power detectors, and FBGs were discussed.

\section{PIEZO-OPTICAL EXPERIMENTAL SETUP}

As shown in Fig 5, A PWAS pitch FBG catch system was set up to validate the system. The software development also reduced a lot of human interaction and provided accurate strain reading instead of voltage reading. The developed FWHM system exhibited good strain sensitivity and an excellent frequency response.

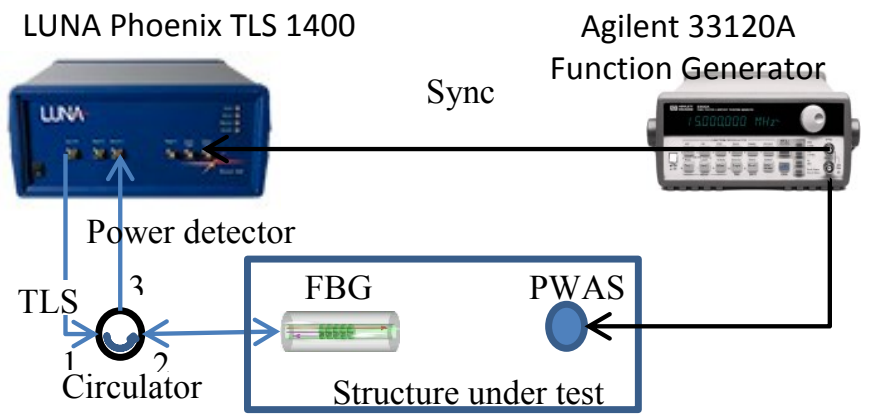

Fig 5: PWAS pitch - FBG catch setup for piezo-optical ultrasonic testing

The measurement equipment for the FBG optical sensor consisted of a LUNA Phoenix 1400 tunable laser source (TLS), an optical circulator (AFW Technologies Pty Ltd, \#CIR-3-15L-1-2), a 50/50 optical splitter (AFW Technologies Pty Ltd, \#FOSC-1-15-50-C-1-S-2), and a PDA10CF photodetector. The output signal from the photodetector was sent to a Tektronix 
TDS5034B digital oscilloscope that also served as signal digitizer.

An Agilent $33120 \mathrm{~A}$ is used to excite PWAS to generate ultrasonic waves in the structure. Since LUNA Phoenix 1400 platform has both tunable laser source and power detectors, it can be served as the hardware for FHMW intensity method. FBG sensor is connected to Phoenix TLS to pick up the strain change in ultrasonic waves. To minimize the human interaction, we developed a software package to automate the whole procedure. The actual strain reading on the FBG is calculated automatically. The magnitude of strain that can be measured is limited by the shape of the FBG spectrum. The frequency response can be very high and is limited only by the response characteristics of the strain transfer to the fiber, the power meter and the sampling instrument.

\section{FBG sensor comparison}

A typical apodized FBG specification from AtGrating Technologies(http://www.atgrating.com/en/productview.asp?id $=63$ ) is shown in Table 1 .

Table 1: Typical FBG specifications (www.atgrating.com)

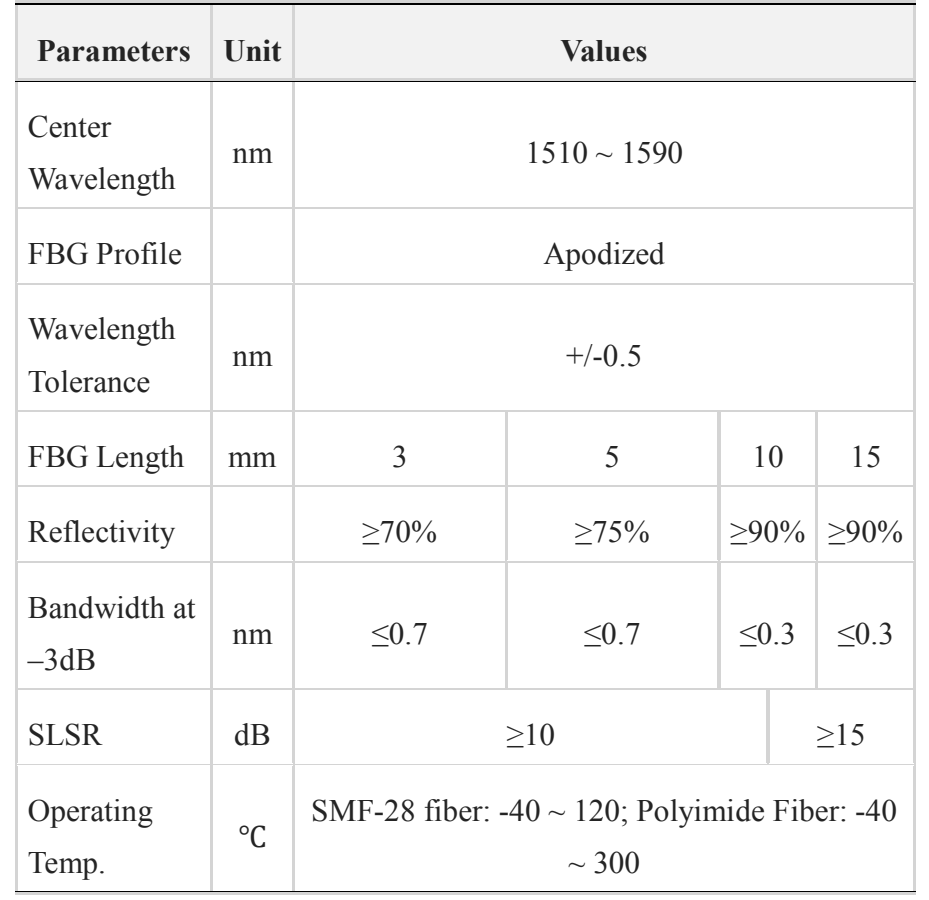

We compared 4 types of FBG sensors available on the market, one from Atgrating, one from Redondo Optics, and two from Micron Optics. The AtGrating FBG was 10-mm long with more than $90 \%$ reflectivity It is made from acrylate fiber (SMF28e) with acrylate recoating. FBG sensor is located at the customized position (1-meter from one FC/APC connector, another 1-meter as pigtail). The FBG is apodized with a center wavelength at $1540 \mathrm{~nm}$. The Redondo Optics FBG was $10-\mathrm{mm}$ long with more than $90 \%$ reflectivity It is also made from acrylate fiber (SMF-28e) with acrylate recoating. FBG sensor location is similar to the Atgrating one (1-meter from one FC/APC connector, another 1-meter as pigtail). The FBG is apodized with a center wavelength at $1550 \mathrm{~nm}$. The two FBG sensors from Micron Optics OS1190 were also 10-mm long. They were made from polyimide fiber with polyimide recoating. The FBG sensors are not apodized to receive the better reflection. The FBG has a wavelength at $1545 \mathrm{~nm}$.

The FBG sensors spectra were shown in Fig 6. Both Atgrating and Redondo FBG sensors were good candidates for FWHM methods since they have smaller side lobes and narrow bandwidth.

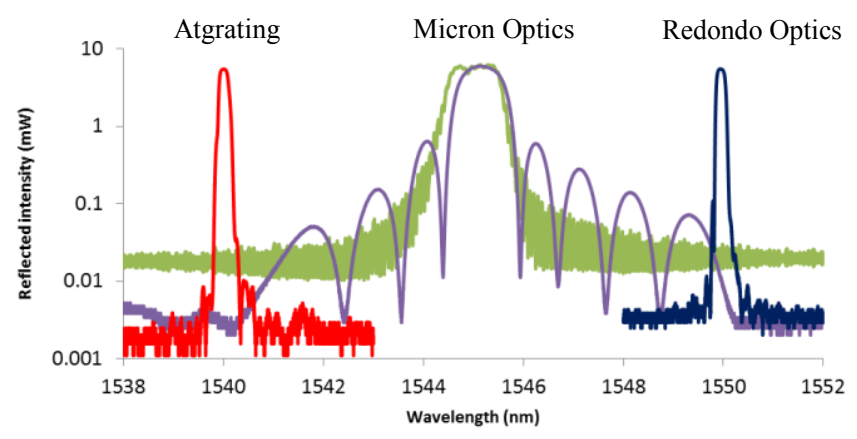

Fig 6: Reflected intensity with different FBG vendors

\section{FBG Strain Measurement Calibration}

The experimental setup for testing the PWAS, FBG sensor, and strain gauge on a steel cantilever beam is shown in Fig 7. The cantilever beam is made of $2.5-\mathrm{mm}$ stainless steel 304 , its dimensions are $609 \mathrm{~mm} \times 18 \mathrm{~mm}$. There are two sensor groups on the beam located at $200-\mathrm{mm}$ and $400-\mathrm{mm}$ from the fixed end. Each sensor group contains one 7-mm square, 0.2-mm thick PZT PWAS, one 10-mm length FBG sensor, and a halfbridge strain gauge. The PWAS and the FBG are bonded backto-back.

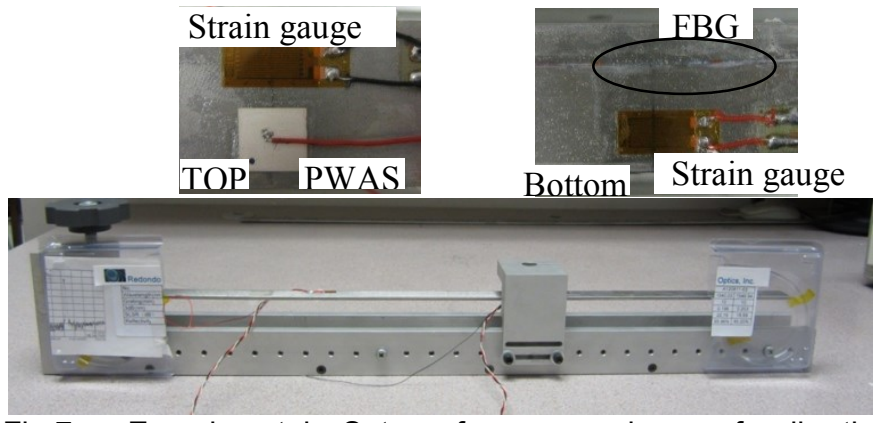

Fig 7 Experimental Setup for comparison of vibration sensing of FBG, strain gauge, and PWAS on a cantilever beam

The FBG strain measurement was calibrated by a static strain measurement. When the cantilever beam was static without any applied force, the strain reading from the optical FBG and strain gauge was set to zero. Then a $10-\mathrm{mm}$ vertical displacement is applied to the beam to apply a static strain on 
the sensor location. The strain was compared with analytical strain calculation, FEM strain simulation, and Vishay strain gauge reading. The fixed vertical displacement yields a static strain, the analytical calculation give the strain value is $33.8 \mu \varepsilon$

. The FEM result is $33.5 \mu \varepsilon$. The strain reading from Vishay strain gauge is $33 \mu \varepsilon$. After conversion, the FBG sensors has a strain reading at $34 \mu \varepsilon$. These results indicate that the strain measurement by FBG agreed well and can be used to calibrate the strain for PWAS dynamic response.

\section{PZT PWAS sensitivity calibrated by a FBG sensor}

The excitation equipment was an HP 33120A signal generator. The excitation signal was a $20-\mathrm{Vpp} 3$-count smoothed tone burst at $300 \mathrm{kHz}$ frequency. The measurement equipment for the FBG sensor was developed and calibrated at LAMSS lab which is able to detect nano-strain changes in $\mathrm{MHz}$ range. The output signal from PWAS receiver was sent to a Tektronix TDS5034B digital oscilloscope that also served as signal digitizer. The strain gauge is connected to a Vishay P4 strain indicator. The 20-Vpp excitation generated an approximate 0.5 $\mu \varepsilon$ peak to peak strain at the excitation location (200-mm from the fixed end) and propagated to the receiver sensor group (400-mm from the fixed end). The strains were picked up by the PWAS and FBG sensor (Fig 8). The strain gauge is not sensitive enough for this application.

(a)

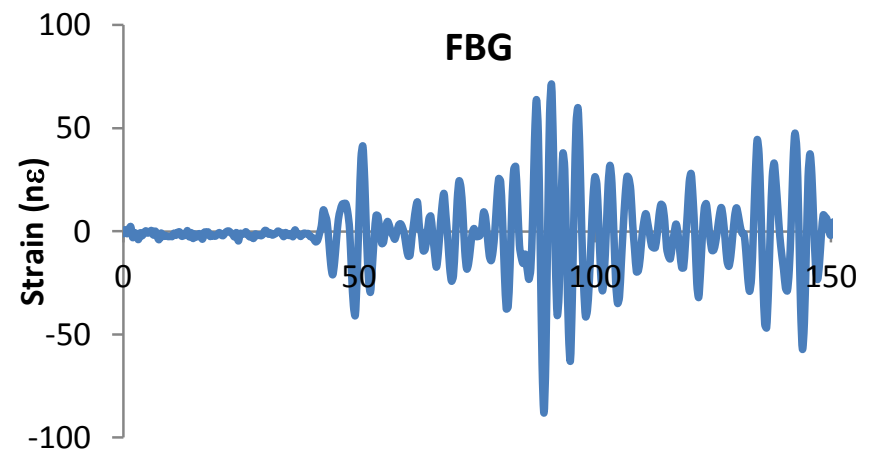

Time ( $\mu \mathrm{s})$

(b)

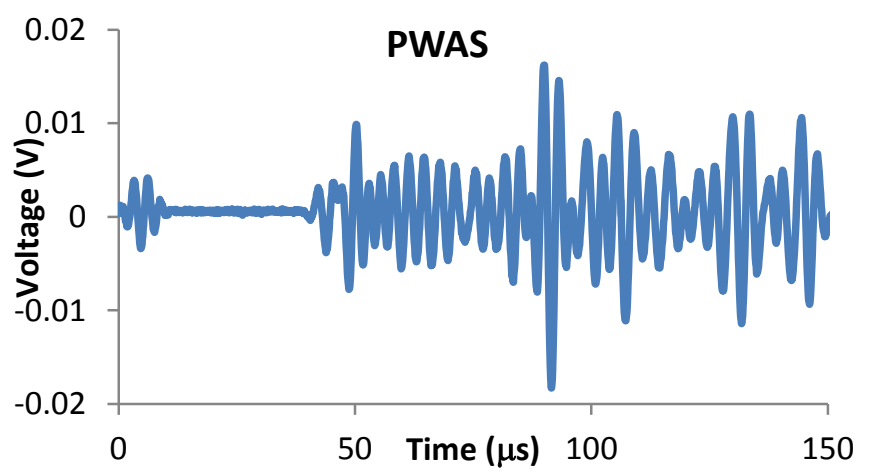

Fig 8 Nano-strain measurement comparison (a) FBG measurement, (b) PWAS measurement
The peak to peak strain detected by the FBG sensor was 0.151 microstrain. The peak to peak voltage generated by the PWAS receiver was 0.032 volt. The PWAS voltage to strain ratio determined by experiment is $0.21 \mathrm{~V} / \mu \varepsilon$.

The simplified equation to calculate the ratio of PWAS strain over voltage is

$$
V / \varepsilon=g_{31} E h
$$

where $E, h, g_{31}$ are the sensor's Young's modulus, its thickness, and the piezoelectric voltage constant. For PZT PWAS at room temperature, the $E$ is $6.3 \times 10^{10} \mathrm{~N} / \mathrm{m}^{2}$ and the $g_{31}$ is $12.4 \times 10^{-3} \mathrm{Vm} / \mathrm{N}$. Using the sensor thickness $(0.2-$ $\mathrm{mm})$, the theoretical ratio is $0.16 \mathrm{~V} / \mu \varepsilon$.

The experimental and theoretical ratios indicate that the PWAS is sensitive to a very small strain. The measurement instrument sensitivity will determine the overall PWAS system sensitivity to strain change without considering the environmental noise. For example, if a data acquisition device (DAQ) has $1 \mathrm{mV}$ resolution, then its strain sensitivity for 0.2 mm PZT PWAS is 5 nanostrain (assume ratio is $0.2 \mathrm{~V} / \mu \varepsilon$ ).

\section{ACOUSTIC EMISSION SENSING}

The detection of acoustic emission (AE) on a cantilever beam specimen included two parts: 1) detection of AE raw signals; 2) detection of $\mathrm{AE}$ events using Mistras PAC equipment.

\section{Acoustic emission signals}

Pencil lead breaks (PLB) are widely used as a reproducible source for test signals in AE applications. AE on the cantilever beam (Fig 7) was simulated by PLB. PWAS and FBG were connected to the oscilloscope to record AE event. Fig 9 shows the raw signals without analog filter and preamplifier which is normally used in the AE data collection. The purpose of the test is to understand how AE propagate through the beam. FBG and PWAS are close to the PLB and used as a trigger for the PLB. Both signals were strong.

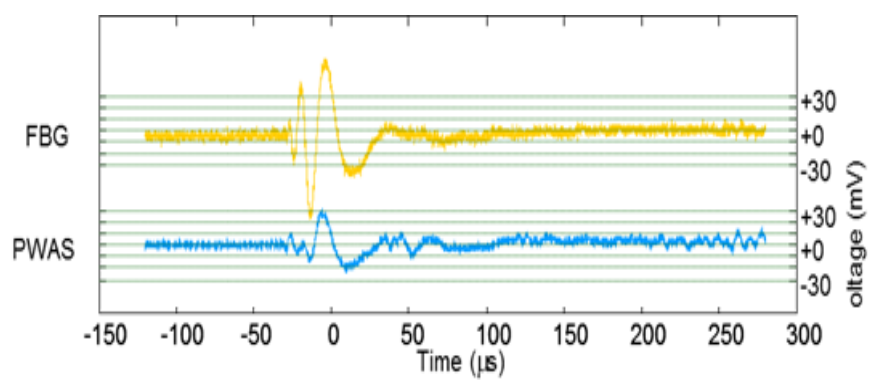

Fig 9 Pencil lead break experiment $A E$ signal of $F B G$ and PWAS

\section{Acoustic emission events}

Both FBG and PWAS were connected to Mistras PAC AE equipment for data acquisition. Each sensor was connected to a 
PAC preamplifier with $100 \mathrm{kHz}-1200 \mathrm{kHz}$ band-pass filter and a fixed $40 \mathrm{~dB}$ gain amplifier. Three PLB events on the surface of the block were recorded. Fig 10 shows the amplitude in $\mathrm{dB}$ of the AE event. Both FBG and PWAS sensors performance were consistent. PWAS has higher amplitude than FBG sensors.

FBG
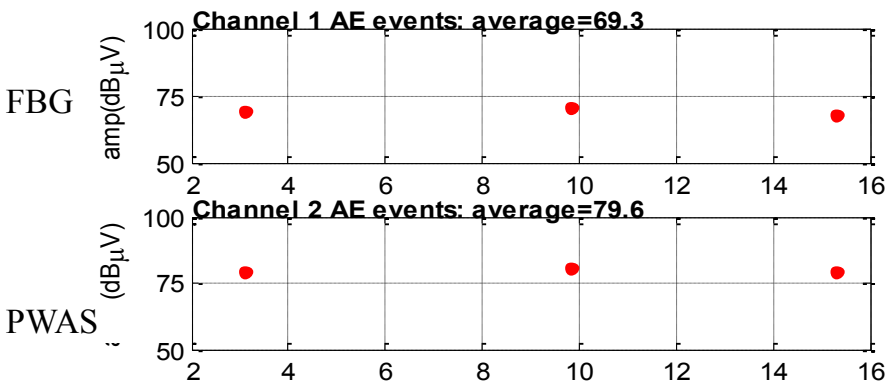

Fig 10 AE event recorded

\section{SUMMARY AND CONCLUSIONS}

This research paper has studied the feasibility of the piezooptical SHM methods. Piezoelectric wafer active sensors (PWAS) and fiber Bragg grating (FBG) sensors were installed onto the specimen and successfully tested. Several flaw detection methods, such as pitch catch and acoustic emission (AE) have been successfully applied. The results obtained in this research have demonstrated the following:

1. In active sensing experiments, PWAS generated wave packets were able to propagate.

2. Both the PWAS receivers and the FBG sensors were able to detect the propagated waves.

3. In passive sensing experiments, simulated acoustic emission sources (pencil lead breaks) were successfully detected with both PWAS and FBG sensors. Both the PWAS transducers and the FBG sensors were able to detect the $\mathrm{AE}$ raw signals directly as well as AE events through the Mistras PAC sensors.

The FBG ultrasonic system was calibrated with a strain gauge under static loading. The dynamic PWAS strain sensing sensitivity wsa calibrated by the FBG sensor. The 0.2-mm PWAS can achieve 5-nanostrain sensitivity with a DAQ resolution of $1 \mathrm{mV}$. The sensitivity can be increased using a thicker PWAS, adding a charge preamplifier, or using a more sensitive voltage measurement device.

\section{ACKNOWLEDGEMENT}

This material is based on work supported by Office of Naval Research \# N00014-11-1-0271 and N00014-12-1-0936, program director Dr. Ignacio Perez. Any opinions, findings, and conclusions or recommendations expressed in this material are those of the authors and do not necessarily reflect the views of the Office of Naval research.

\section{REFERENCE}

[1] Giurgiutiu, V; Gresil, M; Lin, B; Cuc, A; Shen, Y; Roman, C (2012) "Predictive Modeling of Piezoelectric Wafer Active Sensors Interaction with High-frequency Structural Waves and Vibration", Acta Mechanica, March 2012, No. 223, pp. 1681-1691

[2] Balageas, D. et al., (eds.), Structural Health Monitoring, ISTE (2006).

[3] Alleyne, D.N. "The Nondestructive Testing of Plates Using Lamb Waves", Mechanical Engineering Department, Imperial College of London, London, (1991).

[4] Giurgiutiu, V. (2008) Structural Health Monitoring with Piezoelectric Wafer Active Sensors, Elsevier-Academic Press, New York, 2008

[5] Peters, K, "Fiber Bragg Grating Sensors," in Encyclopedia of Structural Health Monitoring, Boller, C;Chang, F-K;Fujino, Y, Eds., ed: Wiley, 2008

[6] Tian, J. J.; Zhang, Q.; Han, M (2013), "Distributed Fiberoptic Laser-Ultrasound Generation based on Ghost Mode of Tilted Fiber Bragg Gratings", Optics Express, Vol. 21, pp. 6109-6114 (2013).

[7] Lin, B.; Giurgiutiu, V. (2014) "Development of optical equipment for ultrasonic guided wave structural health monitoring", SPIE 2014 Smart Structures and NDE, 9-13 March 2014, San Diego, CA, SPIE Vol. 9062, paper \# 906227

[8] Kamal, A.; Lin, B.; Giurgiutiu, V. (2013) "Exact analytical modeling of power and energy for multimode lamb waves excited by piezoelectric wafer active sensors", Journal of Intelligent Material Systems and Structures, Vol. 25, No. 4, pp. $452-471$ 